\title{
A NOTE ON BERNOULLI NUMBERS AND POLYNOMIALS OF HIGHER ORDER
}

\section{CARLITZ}

1. Introduction. Following the notation of Nörlund [5, Chap. 6], we defined $B_{m}^{(k)}, B_{m}^{(k)}(u)$ by means of

$$
\left(\frac{x}{e^{x}-1}\right)^{k} e^{x u}=\sum_{m=0}^{\infty} B_{m}^{(k)}(u) \frac{x^{m}}{m !}, \quad B_{m}^{(k)}=B_{m}^{(k)}(0) \quad(k \geqq 1) .
$$

In the present paper we prove a number of theorems concerning $B_{m}^{(k)}(u)$. It will be convenient to employ the abbreviations

$$
\begin{array}{lll}
(m)_{k}=m(m-1) \cdots(m-k+1), & (m)_{0}=1, \\
{[m]_{k}=\left(a^{m}-1\right)\left(a^{m-1}-1\right) \cdots\left(a^{m-k+1}-1\right),} & {[m]_{0}=1 .}
\end{array}
$$

In the following theorems $p$ denotes an odd prime; the rational numbers $a, u$ are integral $(\bmod p)$ and $p \nmid a$. We now state the following theorems.

THEOREM 1. The number

$$
U_{m}^{(k)}=[m]_{k} B_{m}^{(k)}(u) /(m)_{k} \quad(m \geqq k \geqq 1)
$$

is integral $(\bmod p)$.

THEOREM 2. If $k<p-1, m \neq 0,1, \cdots, k-1(\bmod p-1), m \geqq k$ $\geqq 1$, then $B_{m}^{(k)}(u) /(m)_{k}$ is integral $(\bmod p)$. In particular $B_{m}^{(k)}(u)$ is integral $(\bmod p)$.

THEOREM 3. If $k<p-1, m \neq 0,1, \cdots, k-1(\bmod p-1), m \geqq k$ $\geqq 1, p^{r} \mid(m)_{k}$, then the numerator of $B_{m}^{(k)}(u)$ is divisible by $p^{r}$.

THEOREM 4. Let $U_{m}^{(k)}$ have the same meaning as in (1.3). If $(p-1) p^{e-1} \mid b, m \geqq r b+k, k \geqq 1$, then

$$
\sum_{s=0}^{r}(-1)^{r-s}\left(\begin{array}{l}
r \\
s
\end{array}\right) U_{m+s b}^{(k)} \equiv 0\left(\bmod p^{r e}\right) .
$$

Theorem 5. Put

$$
T_{m}^{(k)}=B_{m}^{(k)}(u) /(m)_{k} \quad(m \geqq k \geqq 1) .
$$

If $k<p-1, m \neq 0,1, \cdots, k-1(\bmod p-1), m \geqq r b+k$, then

Presented to the Society, February 23, 1952; received by the editors November 21, 1951. 


$$
\sum_{s=0}^{r}(-1)^{r-8}\left(\begin{array}{l}
r \\
s
\end{array}\right) T_{m+8 b}^{(k)} \equiv 0\left(\bmod p^{r e}\right)
$$

Theorem 6. If $k<p-1, m \neq 0,1, \cdots, k-1(\bmod p-1), m \geqq r b+k$, $r \geqq k$, then

$$
\sum_{s=0}^{r}(-1)^{r-s}\left(\begin{array}{l}
r \\
s
\end{array}\right) B_{m+s b}^{(k)}(u) \equiv 0\left(\bmod p^{(r-k) e}\right) .
$$

Theorem 7. If $k \leqq p-1, m \equiv s_{0}(\bmod p-1), 0 \leqq s_{0} \leqq k-1$, then

$$
p B_{m}^{(k)}(u) \equiv \frac{(-1)^{k-s_{0}}}{(k-1) !} \frac{(m)_{\dot{k}}}{m-s_{0}}\left(\begin{array}{c}
k-1 \\
s_{0}
\end{array}\right) B_{s_{0}}^{(k)}(u)(\bmod p)
$$

ThEOREM 8. Let $m \equiv s_{0}(\bmod p-1), 0 \leqq s_{0}<p-1$. If $s_{0} \neq 0$, then

$$
p B_{m}^{(p)}(u) \equiv \frac{(m)_{p}}{m-s_{0}} u^{s_{0}}(\bmod p) ;
$$

in particular if $p \mid m-s_{0}$, then

$$
p B_{m}^{(p)}(u) \equiv-u^{s 0} .
$$

However, if $s_{0}=0$, then

$$
p B_{m}^{(p)}(u) \equiv(m)_{p}\left(\frac{1}{m}+\frac{u^{p-1}-1}{m-p+1}\right)(\bmod p) ;
$$

in particular if $p \mid m$, then $p B_{m}^{(p)}(u) \equiv-1$, if $p \mid m+1$, then $p B_{m}^{(p)}(u)$ $\equiv 1-u^{p-1}$.

For references in the case $k=1$, see $[1$, Chap. $1 ; 2 ; 3 ; 4$, Chap. $14 ; 6]$. Vandiver [6] has also discussed the case $k=2$; indeed his numbers of the second order are somewhat more general.

2. Proof of Theorem 1. Let $\eta(x)$ denote a (formal) power series of the type

$$
1+\sum_{1}^{\infty} c_{m}\left(e^{x}-1\right)^{m}
$$

where the $c_{m}$ are integral $(\bmod p)$. Put

$$
g(x)=\left(\frac{x}{e^{x}-1}\right)^{k} \eta(x)
$$

If for brevity we define $\delta^{r} g(x)$ recursively by means of

$$
\delta g(x)=g(a x)-g(x), \quad \delta^{r+1} g(x)=\delta^{r} g(a x)-a^{r} \delta^{r} g(x),
$$


then in the first place, we have

$$
\delta g(x)=\left(\frac{a x}{e^{a x}-1}\right)^{k} \eta(a x)-\left(\frac{x}{e^{x}-1}\right)^{k} \eta(x)=\frac{x^{k}}{\left(e^{x}-1\right)^{k-1}} \eta_{1}(x),
$$

as is easily verified; here $\eta_{1}(x)$ represents a series of the form (2.1). At the next step we find

$$
\begin{aligned}
\delta^{2} g(x) & =\frac{a^{k} x^{k}}{\left(e^{a x}-1\right)^{k-1}} \eta_{1}(a x)-\frac{a x^{k}}{\left(e^{a x}-1\right)^{k-1}} \eta_{1}(x) \\
& =\frac{x^{k}}{\left(e^{x}-1\right)^{k-2}} \eta_{2}(x),
\end{aligned}
$$

where $\eta_{2}(x)$ is also of the form (2.1). Continuing in this way, we finally get

$$
\delta^{k} g(x)=x^{k} \eta_{k}(x),
$$

where of course $\eta_{k}(x)$ is of the form (2.1). Now let $\eta(x)=e^{x_{n}}$ in (2.2); then it is clear from (1.1) that

$$
\frac{\delta^{k} g(x)}{x^{k}}=\sum_{m=k}^{\infty} \frac{[m]_{k} B_{m}^{(k)}(u)}{(m)_{k}} \frac{x^{m-k}}{(m-k) !}=\sum_{m=0}^{\infty} U_{m+k}^{(k)} \frac{x^{m}}{m !} .
$$

Now on the other hand it follows immediately from (2.1) that

$$
\eta(x)=\eta_{k}(x)=\sum_{n=0}^{\infty} b_{n} x^{n} / n !,
$$

where the $b_{n}$ are integral $(\bmod p)$. Comparison with (2.3) and (2.4) yields the theorem.

3. Proof of Theorems 2 and 3. Suppose now that $a$ is a primitive root $(\bmod p)$; then it is clear from the hypothesis of Theorem 2 that none of the factors $a^{k-i}-1, i=0,1, \cdots, k-1$, is divisible by $p$. Consequently $[m]_{k}$ is prime to $p$ and thus Theorem 1 implies Theorem 2.

In the next place, let $p^{r} \mid(m)_{k}$. Since, as we have just seen, $p \nmid[m]_{k}$, it follows from $(1.3)$ that $B_{m}^{(k)}(u) \equiv 0\left(\bmod p^{r}\right)$. Hence Theorem 3 follows.

4. Proof of Theorem 4. We note first that for $\eta(x)$ as defined by (2.1), we have

$$
\eta(x)=1+\sum_{t=1}^{\infty} c_{t} \sum_{s=0}^{t}(-1)^{t-s}\left(\begin{array}{l}
t \\
s
\end{array}\right) \sum_{m=0}^{\infty} \frac{s^{m} x^{m}}{m !} .
$$


Hence if we put

$$
\eta(x)=1+\sum_{m=1}^{\infty} d_{m} x^{m} / m !
$$

it follows that

$$
d_{m}=\sum_{t=1}^{n} c_{t} \sum_{s=0}^{t}(-1)^{t-s}\left(\begin{array}{l}
t \\
s
\end{array}\right) s^{m} \quad(n \geqq m),
$$

since the inner sum in the right member of (4.1) vanishes for $n>m$. Then clearly

$$
\sum_{j=0}^{r}(-1)^{r-j}\left(\begin{array}{l}
r \\
j
\end{array}\right) d_{m+s b}=\sum_{t=1}^{\infty} c_{t} \sum_{s=0}^{t}(-1)^{t-s}\left(\begin{array}{l}
t \\
s
\end{array}\right)(s-1)^{r} s^{m},
$$

where of course the outer sum in the right member is finite. It follows at once that

$$
\sum_{j=0}^{r}(-1)^{r-i}\left(\begin{array}{l}
r \\
j
\end{array}\right) d_{m+j b} \equiv 0\left(\bmod p^{r e}\right)
$$

provided $m \geqq r b$.

Turning now to $U_{m}^{(k)}$, we get from (2.3) and (2.4) that $\delta^{k} g(x) / x^{k}$ is of the form $\eta(x)$ and that the general term in the expansion is of the form $U_{m+k}^{(k)} x^{m} / m !(m \geqq 0)$. Thus we may take $d_{m}=U_{m+k}^{(k)}$, and (4.1) and (4.2) apply. In particular (4.2) implies

$$
\sum_{j=0}^{r}(-1)^{r-j}\left(\begin{array}{l}
r \\
j
\end{array}\right) U_{m+k+j b}^{(k)} \equiv 0\left(\bmod p^{r e}\right)
$$

provided $m \geqq r b$. If we replace $m+k$ by $m$, it is clear that Theorem 4 holds.

5. Proof of Theorem 5. If we substitute from (1.3) in (4.3), we get

$$
\sum_{j=0}^{r}(-1)^{r-j}\left(\begin{array}{l}
r \\
j
\end{array}\right) \frac{[m+j b]_{k} B_{m+j b}^{(k)}(u)}{(m+j b)_{k}} \equiv 0\left(\bmod p^{r e}\right)
$$

provided $m \geqq r b+k$. Suppose now that $a$ is a primitive root $(\bmod p)$ such that $a^{p-1} \equiv 1\left(\bmod p^{w}\right)$ for an arbitrarily assigned $w$. By Theorem 2 we know that $B_{m+j b}^{(k)}(u) /(m+j b)_{k}$ is integral. Hence it suffices to take $w=r e$, so that

$$
[m+j b]_{k} \equiv[m]_{k}\left(\bmod p^{r e}\right) \quad(j=0,1, \cdots, r) .
$$

Thus the left member of (5.1) is congruent to 


$$
[m]_{k} \sum_{j=0}^{r}(-1)^{r-j}\left(\begin{array}{l}
r \\
j
\end{array}\right) B_{m+j b}^{(k)}(u) /(m+j b)_{k}\left(\bmod p^{r e}\right) .
$$

Since $p \nmid[m]_{k},(1.6)$ follows immediately.

6. Proof of Theorem 6. We make use of a device employed by Nielsen [2, Chap. 14]. Let

$$
A_{r, q}=\sum_{s=0}^{r}(-1)^{r-s}\left(\begin{array}{c}
r \\
s
\end{array}\right)\left(\begin{array}{c}
m+s b \\
q
\end{array}\right) T_{m+s b}^{(k)}
$$

so that $A_{r, 0}$ denotes the left member of $(1.6)$ and $A_{r, k}$ the left member of (1.7). We require the recursion

$$
(m+r b-q) A_{r, q}+r b A_{r-1, q}=(q+1) A_{r, q+1},
$$

which is easily verified by substituting from (6.1). Now by the last theorem $A_{r, 0} \equiv 0\left(\bmod p^{r e}\right)$; hence repeated application of (6.2) leads to

$$
A_{r, q} \equiv 0\left(\bmod p^{(r-q) e}\right)
$$

provided $q \leqq r, q<p$. In particular if we take $q=k$ in (6.3), Theorem 6 follows at once.

7. Proof of Theorems 7 and 8. We shall require the following formula $[\mathbf{5}$, p. 148, (87)]:

$$
B_{m}^{(k)}(u)=k\left(\begin{array}{c}
m \\
k
\end{array}\right) \sum_{s=0}^{k-1}(-1)^{k-1-s}\left(\begin{array}{c}
k-1 \\
s
\end{array}\right) \frac{B_{m-s}(u)}{m-s} B_{s}^{(k)}(u),
$$

where $B_{m}(u)=B_{m}^{(1)}(u)$; we also need

$$
\begin{aligned}
& p B_{m}(u) \equiv\left\{\begin{array}{r}
-1 \\
0
\end{array}(\bmod p)\right. \\
& \begin{array}{c}
(p-1 \mid m), \\
(p-1 \nmid m) .
\end{array}
\end{aligned}
$$

Now let $m \equiv s_{0}(\bmod p-1)$, where $0 \leqq s_{0} \leqq k-1$. Since for $s<k$

$$
B_{s}^{(k)}(u)=\frac{s !}{(k-1) !}\left(\frac{d}{d u}\right)^{k-1-s}(u-1)(u-2) \cdots(u-k+1),
$$

it is clear that $B_{s}^{(k)}(u)$ is integral $(\bmod p)$. Thus if we apply (7.2) to the right member of (7.1), we get

$$
p B_{m}^{(k)} \equiv(-1)^{k-s_{0}} k\left(\begin{array}{c}
m \\
k
\end{array}\right)\left(\begin{array}{c}
k-1 \\
s_{0}
\end{array}\right) \frac{B_{s_{0}}{ }^{(k)}(u)}{m-s_{0}}(\bmod p),
$$

which is the same as (1.8). 
To prove Theorem 8 , we again use (7.1). Then for $k=p, s_{0} \neq 0$, it is clear that (7.1) and (7.2) imply

$$
p B_{m}^{(p)}(u) \equiv(-1)^{s_{0}+1} \frac{(m)_{p}}{(p-1) !}\left(\begin{array}{c}
p-1 \\
s_{0}
\end{array}\right) \frac{B_{s_{0}}^{(p)}}{m-s_{0}}(\bmod p) .
$$

Now

$$
\left(\begin{array}{c}
p-1 \\
s
\end{array}\right) \equiv(-1)^{s}
$$

and by (7.3)

$$
B_{s}^{(p)}(u) \equiv \frac{s !}{(p-1) !}\left(\frac{d}{d u}\right)^{p-1-s}\left(u^{p-1}-1\right) \equiv u^{s} \quad(s \leqq p-1) .
$$

Thus

$$
p B_{m}^{(p)}(u) \equiv \frac{(m)_{p}}{m-s_{0}} u^{s_{0}}(\bmod p),
$$

which is identical with (1.9).

As for the case $s_{0}=0$, the only difference is that there are now two terms in (7.1) to consider, namely, those corresponding to $s=0$, $s=p-1$. Thus

$$
p B_{m}^{(p)}(u) \equiv-\frac{(m)_{p}}{(p-1) !}\left(\frac{1}{m}+\frac{1}{m-p+1} B_{p-1}^{(p)}(u)\right)
$$

but by (7.3)

$$
B_{p-1}^{(p)}(u)=(u-1)(u-2) \cdots(u-p+1) \equiv u^{p-1}-1 .
$$

Substitution in (7.4) yields (1.10).

\section{REFERENCES}

1. Paul Bachmann, Niedere Zahlentheorie, vol. 2, Leipzig, 1910.

2. G. Frobenius, Über die Bernoullischen Zahlen und die Eulerschen Polynome, Preuss. Akad. Wiss. Sitzungsber. (1910) pp. 809-847.

3. E. E. Kummer, Über eine allgemeine Eigenschaft der rational Entwickelungscoëfficienten einer bestimmten Gattung analytischer Funktionen, J. Reine Angew. Math. vol. 41 (1951) pp. 368-372.

4. Niels Nielsen, Traité élémentaire des nombres de Bernoulli, Paris, 1923.

5. N. E. Nörlund, Vorlesungen über Differenzenrechnung, Berlin, 1924.

6. H. S. Vandiver, An arithmetical theory of the Bernoulli numbers, Trans. Amer. Math. Soc. vol. 51 (1942) pp. 502-531.

Duke University 